\title{
LOS ACONTECIMIENTOS DEL 11 DE SEPTIEMBRE DE 2001 Y SU RESPUESTA INTERNACIONAL*
}

$\mathrm{El}$ ataque terrorista del 11 de septiembre del año 2001 a Estados Unidos de América, indudablemente violatorio de la normatividad internacional, puso a prueba a la comunidad internacional. El derecho internacional creado por la humanidad a lo largo de su historia tiene una respuesta que puede ser suficiente o no, pero al final de cuentas es una respuesta. Lo importante es si la comunidad internacional da una respuesta racional apegada a derecho ante tal acto de barbarie. Las políticas de fuerza y del talión hace tiempo que están proscritas por la comunidad internacional.

En su esencia, el derecho de gentes es un derecho que busca preservar la paz y la seguridad internacionales; la adecuación a él por parte de la comunidad internacional va a ser sumamente trascendente en el futuro de la humanidad, en el siglo que se inicia, porque el resultado será un fortalecimiento o debilitamiento de la normatividad con el consecuente aumento de las tensiones que llevan a conflictos armados en donde siempre quien pierde es la sociedad civil. Es decir, estos momentos de crisis deben de motivar a la reflexión de los gobernantes de todo el mundo para ver que el derecho internacional es el único elemento que puede hacer posible la convivencia pacífica de los Estados.

Los organismos ONU, OEA, OTAN y el gobierno mexicano se han pronunciado respecto del terrorismo con varias resoluciones que indudablemente serán parte de la historia del derecho internacional, por lo cual aquí las reproducimos.

\footnotetext{
* Nota introductoria a cargo de Manuel Becerra Ramírez.
} 
UNIDOS CONTRA EL TERRORISMO

Kofi ANNAN

Los terroristas que atacaron a Estados Unidos de América el 11 de septiembre tenían como objetivo a una nación pero hirieron al mundo entero. Muy pocas veces, quizás nunca, ha estado el mundo tan unido como lo estuvo en ese día terrible. Era una unidad cimentada en el horror, el miedo, la indignación y la profunda solidaridad con el pueblo de Estados Unidos de América. Esa unidad también nacía del hecho de que en el World Trade Center trabajaban hombres y mujeres de todas las creencias y de más de sesenta naciones. El ataque se había dirigido realmente contra toda la humanidad, y toda la humanidad tiene interés en derrotar a las fuerzas que había detrás de él.

Cuando Estados Unidos de América decidan qué medidas tomarán en defensa de sus ciudadanos y el mundo haga frente a las consecuencias mundiales de esta calamidad, se invocará y se pondrá a prueba la unidad del 11 de septiembre. He expresado al presidente Bush y al alcalde Giuliani $-\mathrm{y}$ a los neoyorquinos en los servicios religiosos celebrados en iglesias, sinagogas y mezquitas - la total solidaridad de las Naciones Unidas con Estados Unidos de América y su pueblo en esta hora de dolor. En menos de 48 horas, el Consejo de Seguridad y la Asamblea General se unieron a mí para condenar los ataques y votaron en apoyo de las medidas que se adopten contra los responsables y contra los Estados que les ayudan, apoyan o protegen. Que nadie ponga en duda esa solidaridad.

Tampoco debe dudar nadie de que el mundo entero está resuelto a luchar contra ese flagelo todo el tiempo que sea necesario. De hecho, la respuesta mundial más elocuente que han recibido hasta ahora los ataques de la semana pasada ha sido el compromiso de los Estados de todas las creencias y todas las regiones a actuar con firmeza contra el terrorismo. En un momento como éste, el mundo se define no sólo afirmándose a favor de algo sino también en contra de algo. Las Naciones Unidas - y la comunidad internacional- deben tener el valor de reconocer que lo mismo que hay objetivos comunes hay enemigos comunes. Para derrotarlos, todas las naciones de buena voluntad deben aunar sus fuerzas en un esfuerzo conjunto que abarque todos los aspectos del sistema mundial abierto y libre que tan arteramente han explotado los autores de las atrocidades de la semana pasada. Las Naciones Unidas ocupan una posición singularmente propicia para promover ese esfuerzo. Ofrecen el foro necesario para formar una coalición universal y pueden dar legitimidad mundial a la reacción contra el terrorismo a largo plazo. Los convenios y convenciones de las Naciones 
Unidas proporcionan ya un marco jurídico para muchas de las medidas que deben adoptarse con el fin de erradicar el terrorismo, incluida la extradición y el enjuiciamiento de los perpetradores, así como la represión del blanqueo de dinero. Es preciso que estos instrumentos se apliquen plenamente.

No obstante, para esa respuesta es esencial que se fortalezca y no se rompa la unidad mundial del 11 de septiembre. Aunque el mundo debe reconocer que hay enemigos comunes a todas las sociedades, debe comprender igualmente que esos enemigos no se definen nunca por sus orígenes religiosos o nacionales. Ningún pueblo, ninguna región y ninguna religión deben ser condenados, atacados ni puestos en la picota por los actos incalificables de unos individuos. Como lo ha dicho el alcalde Giuliani, "eso es exactamente contra lo que luchamos". Él y el presidente Bush han demostrado un admirable liderazgo al condenar los ataques contra los musulmanes en Estados Unidos de América, y otros dirigentes han hecho lo mismo en todo el mundo. Obrar de otro modo y permitir que las divisiones entre las sociedades y dentro de ellas se vean exacerbadas por esos actos sería trabajar en favor de los terroristas, y nadie puede desear ese resultado.

Hoy el terrorismo amenaza a todas las sociedades y a todos los pueblos y cuando el mundo toma medidas contra sus autores, se nos ha recordado a todos la necesidad de afrontar toda la serie de condiciones que permiten el crecimiento de ese odio y esa depravación. Tenemos que oponernos más resueltamente a la violencia, al fanatismo y al odio. Las Naciones Unidas deben continuar su labor mientras combatimos los males de nuestros tiempos - conflictos, ignorancia, pobreza y enfermedad. Con esto no se pondrá fin a todas las fuentes de odio y a todos los actos de violencia- hay quienes odiarán y matarán incluso si se han corregido todas las injusticias. Pero si el mundo puede demostrar que seguirá adelante, que perseverará para crear una comunidad internacional más fuerte, más justa, más generosa y más auténtica por encima de las diferencias de religión y de raza, el terrorismo habrá fracasado. 
SG/SM/7985

AFG/149

8 October 2001

\section{“TO DEFEAT TERRORISM, WE NEED A SUSTAINED EFFORT AND BROAD STRATEGY THAT UNITE ALL NATIONS", SAYS SECRETARY-GENERAL}

Following is the text of a statement made today by Secretary-General Kofi Annan on military strikes in Afghanistan

Immediately after the 11 September attacks on the United States, the Security Council expressed its determination to combat, by all means, threats to international peace and security caused by terrorist acts. The Council also reaffirmed the inherent right of individual or collective self-defence in accordance with the Charter of the United Nations. The States concerned have set their current military action in Afghanistan in that context.

To defeat terrorism, we need a sustained effort and a broad strategy to unite all nations, and address all aspects of the scourge we face. The cause must be pursued by all the States of the world, working together and using many different means - including political, legal, diplomatic and financial means-.

The people of Afghanistan, who cannot be held responsible for the acts of the Taliban regime, are now in desperate need of aid. The United Nations has long played a vital role in providing humanitarian assistance to them, and it is my hope that we will be able to step up our humanitarian work as soon as possible.

It is also vital that the international community now work harder than ever to encourage a political settlement to the conflict in Afghanistan. The United Nations is actively engaged in promoting the creation of a fully representative, multi-ethnic and broad-based Afghan Government.

\section{El Consejo de SEguridad de la ONU CONDENA LOS ATAQUES TERRORISTAS EN ESTADOS UNIDOS}

Declaración del presidente del Consejo de Seguridad de las Naciones Unidas, Jean-David Levitte (Francia) condenando los ataques terroristas perpetrados en Estados Unidos de América

Los miembros del Consejo de Seguridad están conmocionados por los espantosos ataques terroristas perpetrados hoy en Nueva York, Washington D. C., y en otros sitios de Estados Unidos de América, y los condenan uná- 
nimemente de la manera más categórica. Esta es una tragedia y un reto para toda la humanidad. No puede haber excusa ni justificación para estos actos ni para cualquier acto terrorista.

Los miembros del Consejo de Seguridad expresan sus más profundas condolencias a las víctimas y a sus familias, así como al pueblo y al gobierno de Estados Unidos de América.

Los miembros del Consejo de Seguridad hacen un llamado a todos los Estados para trabajar en conjunto y urgentemente para llevar ante la justicia a los perpetradores, organizadores y auspiciadores de las atrocidades cometidas hoy. Los miembros del Consejo de Seguridad hacen un llamado a la comunidad internacional para redoblar sus esfuerzos en la prevención y eliminación de los actos terroristas mediante una mayor cooperación y el estricto cumplimiento de las convenciones internacionales contra el terrorismo y de las resoluciones del Consejo de Seguridad.

Los miembros del Consejo de Seguridad expresan su disposición para tomar medidas urgentes de conformidad con las responsabilidades que les atribuye la Carta de las Naciones Unidas.

\section{EL SECRETARIO GENERAL DE LAS NACIONES UNIDAS CONDENA LOS ATAQUES TERRORISTAS EN ESTADOS UNIDOS DE AMÉRICA}

Declaración del secretario general de las Naciones Unidas, Kofi Annan, sobre los ataques perpetrados hoy en Nueva York

Todos hemos sido traumatizados por esta terrible tragedia. Aún no sabemos cuántas personas han muerto o han sido heridas, pero sin duda será un número alto. Nuestros primeros pensamientos y oraciones deben ser para las víctimas y sus familiares. Deseo expresar mis profundas condolencias a todos ellos, al pueblo y al gobierno de Estados Unidos de América.

No cabe duda de que estos ataques han sido deliberados actos de terrorismo, planeados y coordinados cuidadosamente. Como tales, los condeno completamente. El terrorismo debe ser combatido con determinación donde quiera que surja.

En estos momentos, es más importante que nunca el razonamiento con calma y cordura. Aún no sabemos quién está detrás de estos actos o qué objetivo persiguen. Lo que sí sabemos es que ninguna causa justa puede ser impulsada por medio del terror. 


\section{CONSEJO DE SEGURIDAD}

S/RES/1269 (1999)

19 de octubre de 1999

RESOLUCIÓN 1269 (1999)

Aprobada por el Consejo de Seguridad en su 40532 sesión, celebrada el 19 de octubre de 1999

El Consejo de Seguridad,

Profundamente preocupado por el aumento de los actos de terrorismo internacional que ponen en peligro la vida y el bienestar de las personas en todo el mundo, así como la paz y la seguridad de todos los Estados,

Condenando todos los actos terroristas, independientemente de su motivación y dondequiera y por quienquiera que sean cometidos,

Consciente de todas las resoluciones pertinentes de la Asamblea General, incluida la resolución 49/60, de 9 de diciembre de 1994, por la que la Asamblea aprobó la Declaración sobre Medidas para Eliminar el Terrorismo Internacional,

Subrayando la necesidad de intensificar la lucha contra el terrorismo en el plano nacional y de reforzar, bajo los auspicios de las Naciones Unidas, la cooperación internacional efectiva en esta esfera basada en el respeto de los principios de la Carta de las Naciones Unidas y las normas del derecho internacional, incluido el respeto del derecho internacional humanitario y de los derechos humanos,

Apoyando los esfuerzos encaminados a promover la participación universal en las convenciones internacionales vigentes contra el terrorismo y su aplicación, así como a elaborar nuevos instrumentos internacionales para hacer frente a la amenaza terrorista,

Encomiando la labor desarrollada por la Asamblea General, los órganos y los organismos especializados pertinentes de las Naciones Unidas y las organizaciones regionales y de otro tipo para luchar contra el terrorismo internacional,

Decidido a contribuir, de conformidad con la Carta de las Naciones Unidas, a los esfuerzos encaminados a luchar contra toda forma de terrorismo,

Reafirmando que la represión de los actos de terrorismo internacional, incluidos aquellos en los que están implicados los Estados, es una contribución esencial al mantenimiento de la paz y la seguridad internacionales,

1. Condena inequívocamente todos los actos, métodos y prácticas terroristas por considerarlos criminales e injustificables, independientemente de 
su motivación y dondequiera y por quienquiera que sean cometidos, en todas sus formas y manifestaciones, en particular los que puedan representar una amenaza para la paz y la seguridad internacionales;

2. Hace un llamamiento a todos los Estados para que apliquen plenamente las convenciones internacionales contra el terrorismo en las que son partes, alienta a todos los Estados a considerar con carácter prioritario la posibilidad de adherirse a aquéllas en las que no lo son y alienta también a que se aprueben con rapidez las convenciones pendientes;

3. Destaca la función vital que desempeñan las Naciones Unidas en el fortalecimiento de la cooperación internacional en la lucha contra el terrorismo y pone de relieve la importancia de una mayor coordinación entre los Estados y las organizaciones internacionales y regionales;

4. Hace un llamamiento a todos los Estados para que, entre otras cosas, en el contexto de esa cooperación y coordinación, adopten medidas apropiadas para:

Cooperar entre sí, especialmente en el marco de acuerdos y arreglos bilaterales y multilaterales, para prevenir y reprimir los actos terroristas, proteger a sus nacionales y otras personas de los ataques terroristas y enjuiciar a los responsables de tales actos;

Prevenir y reprimir en sus territorios por todos los medios lícitos la preparación y financiación de todo acto terrorista;

Denegar refugio a quienes planifiquen, financien o cometan actos terroristas, velando por que sean detenidos y procesados o extraditados;

Cerciorarse, antes de conceder el estatuto de refugiado a quien solicite asilo, de conformidad con las disposiciones pertinentes del derecho nacional e internacional, incluidas las normas internacionales de derechos humanos, de que no haya participado en actos terroristas;

Intercambiar información, de conformidad con el derecho internacional y nacional, y cooperar en asuntos administrativos y judiciales con el fin de prevenir la comisión de actos terroristas;

5. Pide al secretario general que, en los informes que presente a la Asamblea General, en particular en virtud de lo dispuesto en su resolución 50/53 sobre la adopción de medidas destinadas a eliminar el terrorismo internacional, preste atención especial a la necesidad de prevenir y combatir la amenaza que las actividades terroristas representan para la paz y la seguridad internacionales;

6. Expresa que está [sic] de los informes mencionados en el párrafo 5 supra y a adoptar las medidas necesarias de conformidad con la responsabilidad que le incumbe en virtud de la Carta de las Naciones Unidas, para hacer frente a las amenazas terroristas y la seguridad internacional.

7. Decide seguir ocupándose de esta cuestión. 
RESOLUCIÓN 1333 (2000)

S/RES/1333 (2000)

Distr. general

19 de diciembre de 2000

00-80665 (S)

Aprobada por el Consejo de Seguridad en su 4251 sesión, celebrada el 19 de diciembre de 2000

El Consejo de Seguridad,

Reafirmando sus resoluciones anteriores, en particular la resolución 1267 (1999), de 15 de octubre de 1999, así como las declaraciones de su presidente acerca de la situación en el Afganistán,

Reafirmando su firme apoyo a la soberanía, la independencia, la integridad territorial y la unidad nacional de Afganistán, así como su respeto al patrimonio cultural e histórico del país,

Reconociendo las necesidades humanitarias críticas del pueblo afgano, Respaldando las gestiones que realiza el representante personal del secretario general para el Afganistán para impulsar un proceso de paz recurriendo a negociaciones políticas entre las partes afganas con miras al establecimiento de un gobierno de amplia base, pluriétnico y plenamente representativo, y haciendo un llamamiento a las partes beligerantes para que colaboren plenamente en esas gestiones para que se llegue a una cesación del fuego y se emprendan conversaciones encaminadas a lograr un acuerdo político, avanzando prontamente en el proceso de diálogo al que se han comprometido,

Tomando nota de la reunión celebrada en diciembre de 2000 por el Grupo de Apoyo para el Afganistán, el que subrayó que la situación en el Afganistán era compleja y exigía un enfoque general e integrado sobre un proceso de paz y las cuestiones del tráfico de estupefacientes, el terrorismo, los derechos humanos y la ayuda internacional humanitaria y para el desarrollo,

Recordando las convenciones internacionales pertinentes contra el terrorismo y, en particular, las obligaciones de las partes en esas convenciones de extraditar o procesar a los terroristas,

Condenando enérgicamente el persistente uso de las zonas del Afganistán dominadas por la facción afgana conocida como los talibanes, que se denomina a sí misma Emirato Islámico del Afganistán (en lo sucesivo los talibanes) para dar refugio y entrenar a terroristas y planificar actos de terrorismo, y reafirmando su convencimiento de que la supresión del terroris- 
mo internacional es imprescindible para el mantenimiento de la paz y la seguridad internacionales,

Observando la importancia de que los talibanes actúen de conformidad con la Convención única de 1961, el Convenio sobre Sustancias Sicotrópicas de 1971, la Convención de las Naciones Unidas contra el Tráfico Ilícito de Estupefacientes y Sustancias Sicotrópicas de 1988 y los compromisos del vigésimo periodo extraordinario de sesiones de la Asamblea General, celebrado en 1998 para examinar el problema de los estupefacientes, que incluyen colaborar estrechamente con el Programa de las Naciones Unidas para la Fiscalización Internacional de Drogas,

Tomando nota de que los talibanes se benefician directamente del cultivo ilícito de opio mediante la imposición de un gravamen sobre su producción y de que se benefician indirectamente del procesamiento y el tráfico ilícitos de opio, y reconociendo que esos recursos sustanciales aumentan la capacidad de los talibanes de dar acogida a los terroristas,

Deplorando el hecho de que los talibanes sigan proporcionando un refugio seguro a Usama bin Laden y permitiendo que él y sus asociados dirijan una red de campamentos de entrenamiento de terroristas en el territorio controlado por los talibanes y que utilicen al Afganistán como base para patrocinar operaciones terroristas internacionales,

Tomando nota del auto de acusación de Usama bin Laden y sus asociados por Estados Unidos de América, entre otras cosas, por la colocación de bombas en las embajadas de ese país en Nairobi (Kenya) y Dar es Salam (República Unida de Tanzania) el 7 de agosto de 1998 y por conspirar contra la vida de ciudadanos norteamericanos fuera de Estados Unidos de América, y tomando nota asimismo de la petición de Estados Unidos de América a los talibanes de que los entreguen para que sean procesados (S11999/1021),

Reiterando su profunda preocupación por el hecho de que continúen las violaciones del derecho internacional humanitario y de los derechos humanos, en particular la discriminación contra las mujeres y las niñas, así como por el aumento considerable de la producción ilícita de opio,

Subrayando que la ocupación por los talibanes del Consulado General de la República Islámica del Irán y el asesinato de diplomáticos iraníes y de un periodista en Mazar-e-Sharif constituyen violaciones manifiestas de las normas establecidas de derecho internacional,

Habiendo determinado que el hecho de que las autoridades de los talibanes no hayan respondido a las exigencias formuladas en el párrafo 13 de la resolución 1214 (1998) y el párrafo 2 de la resolución 1267 (1999) constituye una amenaza para la paz y la seguridad internacionales,

Destacando su determinación de hacer respetar sus resoluciones, 
Reafirmando la necesidad de que las sanciones contengan exenciones suficientes y eficaces para evitar que se produzcan consecuencias humanitarias perjudiciales para la población del Afganistán, y de que se estructuren de manera que no obstaculicen, frustren ni retrasen la labor de las organizaciones internacionales de asistencia humanitaria y los organismos gubernamentales de socorro que prestan asistencia humanitaria a la población del país,

Subrayando que los talibanes son responsables del bienestar de la población en las zonas del Afganistán que están dominadas por ellos y, a este respecto, haciendo un llamamiento a los talibanes para que garanticen el acceso en condiciones de seguridad y sin trabas del personal y la ayuda de socorro a todas las personas necesitadas que se encuentran en el territorio que dominan,

Recordando los principios pertinentes de la Convención sobre la Seguridad del Personal de las Naciones Unidas y el Personal Asociado, aprobada por la Asamblea General en su resolución 49159, de 9 de diciembre de 1994 ,

Actuando en virtud del capítulo VII de la Carta de las Naciones Unidas,

1. Exige que los talibanes cumplan con lo dispuesto en la resolución 1267 (1999) y, en particular, dejen de dar refugio y de entrenar a terroristas internacionales y a sus organizaciones, tomen medidas eficaces y apropiadas para que no se use el territorio que dominan para albergar instalaciones y campamentos de terroristas ni para la preparación u organización de actos de terrorismo contra otros Estados o sus ciudadanos, y colaboren en las actividades internacionales encaminadas a poner a disposición de los tribunales a las personas acusadas de delitos de terrorismo;

2. Exige también que los talibanes den cumplimiento sin demora a lo dispuesto en el párrafo 2o. de la resolución 1267 (1999) del Consejo de Seguridad, en que se exige que los talibanes entreguen a Usama bin Laden a las autoridades competentes de un país donde haya sido objeto de un auto de acusación, a las autoridades competentes de un país desde el cual haya de ser devuelto al primero o a las autoridades competentes de un país donde haya de ser detenido y debidamente enjuiciado;

3. Exige además que los talibanes procedan rápidamente a clausurar todos los campamentos en que se entrene a terroristas dentro del territorio que dominan, y piden que las Naciones Unidas confirmen esas clausuras haciendo uso de la información que les faciliten los Estados miembros de conformidad con lo dispuesto en el párrafo 19 infra o de otras medidas que sean necesarias para garantizar el cumplimiento de la presente resolución;

4. Recuerda a todos los Estados su obligación de dar estricto cumplimiento a las medidas impuestas en el párrafo 4 de la resolución 1267 (1999); 
5. Decide que todos los Estados:

a) Impedirán el suministro, la venta y la transferencia directa o indirecta al territorio del Afganistán dominado por los talibanes que indique el comité establecido en virtud de la resolución 1267 (1999), en lo sucesivo denominado el comité, por sus nacionales o desde sus territorios o utilizando buques o aeronaves de su pabellón, de armas y materiales conexos de todo tipo, incluidas las armas y municiones, los vehículos y pertrechos militares, los pertrechos paramilitares y las piezas de repuesto correspondientes;

b) Impedirán la venta, el suministro y la transferencia directa o indirecta al territorio del Afganistán dominado por los talibanes que indique el comité, por sus nacionales o desde sus territorios, de servicios de asesoramiento, asistencia o capacitación relacionados con las actividades militares del personal armado bajo el control de los talibanes;

c) Retirarán a todos sus oficiales, agentes, asesores, y militares empleados mediante contrato u otro tipo de acuerdo que se hallen en el Afganistán para asesorar a los talibanes acerca de asuntos militares o de seguridad conexos; e instarán a otros nacionales en este contexto a que abandonen el país;

6. Decide que las medidas establecidas en el párrafo 5 supra no se aplicarán a los suministros de equipo militar no mortífero destinado exclusivamente a fines humanitarios y de protección y la asistencia o capacitación técnica conexa que haya aprobado previamente el comité, y afirma asimismo que las medidas impuestas en el párrafo 5 supra no se aplicarán al vestuario de protección, incluidos los chalecos a prueba de balas y los cascos militares exportados al Afganistán por el personal de las Naciones Unidas, representantes de los medios de comunicación y trabajadores humanitarios exclusivamente para su uso particular,

7. Insta a todos los Estados que mantengan relaciones diplomáticas con los talibanes a que reduzcan considerablemente el número y la categoría de los funcionarios en misiones y puestos de los talibanes y restrinjan o controlen los desplazamientos por su territorio de todo el personal que permanezca allí; en el caso de las misiones de los talibanes ante organizaciones internacionales, el Estado anfitrión podrá, si lo juzga necesario, consultar a la organización que corresponda en relación con las medidas que hayan de adoptarse para aplicar este párrafo;

8. Decide que todos los Estados adopten nuevas medidas para:

a) Clausurar inmediata y completamente todas las oficinas de los talibanes en sus territorios;

b) Clausurar inmediatamente todas las oficinas de Ariana Afghan Airlines en sus territorios,

c) Congelar sin demora los fondos y otros activos financieros de Usama bin Laden y de las personas y entidades con él asociados indicados por el 
comité, incluidos los de la organización Al-Qaida y los fondos dimanantes u obtenidos de bienes poseídos o controlados directa o indirectamente por Usama bin Laden y las personas y entidades con él asociados, y velar por que esos u otros fondos o recursos financieros no sean utilizados, directa o indirectamente, por sus nacionales o por personas que se hallen en su territorio en beneficio de Usama bin Laden, las personas asociadas con él o entidades poseídas o controladas directa o indirectamente por Usama bin Laden o personas o entidades con él asociadas, incluida la organización Al-Qaida, y pide al comité que mantenga una lista actualizada, basada en información suministrada por los Estados y organizaciones regionales, de las personas y entidades que se haya indicado que están asociadas con Usama bin Laden, incluidas las de la organización Al-Qaida;

9. Exige que los talibanes y otros grupos pongan fin a todas las actividades ilegales relacionadas con los estupefacientes y procedan a eliminar prácticamente el cultivo ilícito de adormidera para la producción de opio, con cuyas ganancias se financian las actividades terroristas de los talibanes;

10. Decide que todos los Estados impidan la venta, el suministro o la transferencia por sus nacionales o desde sus territorios del producto químico anhídrido acético a todas las personas que se hallen en el territorio del Afganistán dominado por los talibanes que indique el comité, y a todas las personas a los efectos de la realización de actividades en el territorio del Afganistán dominado por los talibanes que indique el comité, o desde él;

11. Decide asimismo que todos los Estados deberán negar la autorización de despegar desde su territorio, de aterrizar en él o de sobrevolarlo a cualquier aeronave que haya despegado del territorio del Afganistán que el comité haya indicado que se encuentra dominado por los talibanes o tenga previsto aterrizar en él, salvo que el vuelo de que se trate haya sido aprobado de antemano por el comité por razones de necesidad humanitaria, incluido el cumplimiento de una obligación religiosa, como el Hadj, o se considere que el vuelo sirve para promover la negociación de una solución pacífica del conflicto en el Afganistán o sea probable que promueva el cumplimiento por los talibanes de la presente resolución o de la resolución 1267 (1999);

12. Decide además que el comité lleve una lista de las organizaciones y los organismos gubernamentales de socorro aprobados que prestan asistencia humanitaria al Afganistán, incluidos las Naciones Unidas y sus organismos, los organismos gubernamentales de socorro que presten asistencia humanitaria, el Comité Internacional de la Cruz Roja y las organizaciones no gubernamentales, según proceda, que la prohibición que impone el párrafo 11 supra no será aplicable a los vuelos humanitarios que hagan las organizaciones y los organismos gubernamentales de socorro, incluidos en la lista aprobada por el comité o que se hagan en nombre de ellos, que el comité 
tenga la lista en examen constante, añadiendo organizaciones y organismos gubernamentales de socorro según proceda, y que el comité suprima organizaciones y organismos gubernamentales de la lista si decide que están haciendo o es probable que hagan vuelos con fines no humanitarios y notificará inmediatamente a tales organizaciones y organismos gubernamentales que los vuelos que hagan o que se hagan en su nombre estarán por tanto sujetos a lo dispuesto en el párrafo 11 supra;

13. Hace un llamamiento a los talibanes para que garanticen el acceso en condiciones de seguridad y sin trabas del personal y la ayuda de socorro a todas las personas necesitadas que se encuentran en el territorio que dominan, y subraya que los talibanes han de garantizar la protección, la seguridad y la libertad de circulación del personal de las Naciones Unidas que presta socorro humanitario y el personal conexo;

14. Insta a los Estados a que tomen medidas para restringir la entrada en su territorio o el paso por él de todos los funcionarios superiores de los talibanes con rango de viceministro o rangos superiores, del personal armado con rango equivalente bajo el control de los talibanes y de otros asesores superiores y dignatarios de los talibanes, a menos que dichos funcionarios viajen con fines humanitarios, incluido el cumplimiento de alguna obligación religiosa, como el Hadj, o el viaje promueva la negociación de una solución pacífica del conflicto del Afganistán o se haga en cumplimiento de la presente resolución o de la resolución 1267 (1999);

15. Pide al secretario general que, en consulta con el comité:

a) Nombre un comité de expertos para que haga recomendaciones al consejo dentro de los 60 días siguientes a la aprobación de la presente resolución sobre el modo de vigilar el embargo de armas y la clausura de los campamentos de entrenamiento de terroristas exigidos en los párrafos 3 y 5 supra, incluso, entre otras cosas, el uso de la información que obtengan los Estados miembros por sus medios nacionales y que suministren al secretario general;

b) Consulte a los Estados miembros que corresponda para aplicar las medidas enunciadas en la presente resolución y en la resolución 1267 (1999) y le informe de los resultados de esas consultas,

c) Le presente un informe sobre el cumplimiento de las medidas vigentes, evalúe los problemas relacionados con la aplicación de esas medidas, haga recomendaciones para reforzar esa aplicación y evalúe las medidas que adopten los talibanes para cumplir las condiciones;

d) Examine las consecuencias humanitarias de las medidas impuestas en la presente resolución y en la resolución 1267 (1999), y presente un informe al consejo dentro de un plazo de 90 días contados a partir de la aprobación de la presente resolución, acompañado de una evaluación y de recomendaciones, y que posteriormente presente periódicamente otros sobre las 
consecuencias humanitarias, y le presente un informe general sobre esta cuestión, así como recomendaciones a más tardar 30 días después de que se ponga fin a esas medidas;

16. Pide al comité que, en cumplimiento de su mandato, realice las siguientes tareas además de las enunciadas en la resolución 1267 (1999):

a) Prepare y lleve listas actualizadas, basadas en la información que suministren los Estados y las organizaciones regionales e internacionales, de todos los puntos de entrada y zonas de aterrizaje de aeronaves dentro del territorio del Afganistán dominado por los talibanes y comunique a los Estados miembros el contenido de dichas listas;

b) Prepare y lleve listas actualizadas, basadas en la información que suministren los Estados y las organizaciones regionales, de las personas y entidades que se haya indicado que están asociadas con Usama bin Laden, de conformidad con el apartado c) del párrafo 8 supra;

c) Examine y resuelva las solicitudes de que se concedan las excepciones enunciadas en los párrafos 6 y 11 supra;

d) Prepare y lleve, antes de que transcurra un mes desde la aprobación de la presente resolución, una lista actualizada de las organizaciones y los organismos gubernamentales de socorro autorizados que presten asistencia humanitaria al Afganistán, de conformidad con el párrafo 12 supra;

e) Publique información pertinente relativa a la aplicación de estas medidas por medios adecuados, incluso mediante una mejor utilización de la tecnología de la información;

f) Considere la posibilidad, cuando proceda, de que el presidente del comité y los otros miembros que sean necesarios para la aplicación plena y eficaz de las medidas impuestas en la presente resolución y en la resolución 1267 (1999) se desplacen a los países de la región para instar a los Estados a que cumplan las resolución pertinentes del consejo;

g) Presente informes periódicos al consejo con la información que se le facilite en relación con esta resolución y la resolución 1267 (1999), incluidas las eventuales violaciones de las medidas que se comuniquen al comité $\mathrm{y}$ recomendaciones para el fortalecimiento de la eficacia de las medidas,

17. Exhorta a todos los Estados y a todas las organizaciones internacionales y regionales, incluso las Naciones Unidas y sus organismos especializados, a que actúen estrictamente de conformidad con lo dispuesto en la presente resolución, no obstante la existencia de derechos u obligaciones reconocidos o impuestos por acuerdos internacionales o contratos o licencias o permisos concedidos antes de la fecha de entrada en vigor de las medidas impuestas en los párrafos 5, 8, 10 y 11 supra;

18. Exhorta a los Estados a que procesen a las personas y entidades que estén bajo su jurisdicción y hayan violado las medidas impuestas en los párrafos 5, 8, 10 y 11 supra y a que les impongan penas adecuadas, 
19. Exhorta también a todos los Estados a que colaboren plenamente con el comité en el cumplimiento de sus tareas, incluido el suministro de la información que requiera el comité en cumplimiento de la presente resolución,

20. Pide a todos los Estados que informen al comité, dentro de los 30 días siguientes a la entrada en vigor de las medidas impuestas en los párrafos $5,8,10$ y 11 supra, acerca de las disposiciones que hayan tomado con miras a cumplir eficazmente la resolución;

21. Pide a la secretaria que someta a la consideración del comité la información que reciba de los gobiernos y de fuentes públicas sobre eventuales violaciones de las medidas impuestas en los párrafos 5, 8, 10 y 11 supra;

22. Decide que las medidas impuestas en los párrafos 5, 8, 10 y $11 \mathrm{su}$ pra en tren en vigor a las 00.01 horas (hora de Nueva York) al cabo de un mes de la aprobación de la presente resolución;

23. Decide también que las medidas impuestas en los párrafos 5, 8, $10 \mathrm{y}$ 11 supra estén en vigor durante 12 meses y que, al final de ese periodo, el consejo decida si los talibanes han cumplido o no lo dispuesto en los párrafos 1,2 y 3 supra y, en consecuencia, si prorroga esas medidas por un nuevo periodo con las mismas condiciones;

24. Decide, además, que si los talibanes cumplen las condiciones enunciadas en los párrafos 1, 2 y 3 supra, antes de que haya transcurrido el periodo de 12 meses, el consejo pondrá fin a las medidas impuestas en los párrafos 5, 8, 10 y 11 supra;

25. Expresa su disposición a considerar la posibilidad de imponer otras medidas, de conformidad con la responsabilidad que le asigna la Carta de las Naciones Unidas, con el fin de lograr el pleno cumplimiento de la presente resolución y de la resolución 1267 (1999), teniendo en cuenta, entre otras cosas, la evaluación de las consecuencias que se indica en el apartado d) del párrafo 15, a los efectos de acrecentar la eficacia de las sanciones y reducir al mínimo las consecuencias negativas en la situación humanitaria,

26. Decide seguir ocupándose activamente de la cuestión.

\section{RESOLUCIÓN 1363 (2001)}

Distr. general

30 de julio de 2001

Aprobada por el Consejo de Seguridad en su 4352 sesión, celebrada el 30 de julio de 2001

El Consejo de Seguridad,

Reafirmando sus resoluciones anteriores, en particular sus resoluciones 1267 (1999), de 15 de octubre de 1999, y 1333 (2000), de 19 de diciembre 
de 2000, así como las declaraciones de su presidente acerca de la situación en el Afganistán,

Determinando que la situación en el Afganistán constituye una amenaza para la paz y la seguridad internacionales en la región,

Actuando en virtud del capítulo VII de la Carta de las Naciones Unidas,

1. Subraya la obligación que incumbe a todos los Estados miembros en virtud de la Carta de las Naciones Unidas de dar cumplimiento estricto a las medidas impuestas por las resoluciones 1267 (1999) y 1333 (2000);

2. Acoge con beneplácito el informe (S/2001/51 1) del Comité de Expertos establecido en virtud de la resolución 1333 (2000), y observa las conclusiones y recomendaciones que en él figuran, hechas tras las consultas con los Estados que limitan con el territorio del Afganistán controlado por los talibanes que había visitado;

3. Pide al secretario general que, en consulta con el comité establecido en virtud de la resolución 1267 (1999), establezca, dentro de un plazo de 30 días de la fecha de aprobación de la presente resolución y durante un periodo simultáneo al de la aplicación de las medidas impuestas por la resolución 1333 (2000), un mecanismo encargado de:

a) Vigilar la aplicación de las medidas impuestas por las resoluciones 1267 (1999) y 1333 (2000);

b) Ayudar a los Estados que limitan con el territorio del Afganistán controlado por los talibanes y a otros Estados, según proceda, a incrementar su capacidad para aplicar las medidas impuestas por las resoluciones 1267 (1999) y 1333 (2000), y

c) Reunir, evaluar, verificar cuando sea posible y comunicar información relativa a violaciones de las medidas impuestas por las resoluciones 1267 (1999) y 1333 (2000), y hacer recomendaciones al respecto;

4. Decide que, teniendo en cuenta entre otras cosas una distribución geográfica equitativa, el mecanismo de vigilancia esté integrado por:

a) Un Grupo de Vigilancia en Nueva York de hasta cinco expertos, incluido un presidente, para vigilar la aplicación de todas las medidas impuestas por las resoluciones 1267 (1999) y 1333 (2000), incluso en materia de embargo de armas, medidas contra el terrorismo y legislación conexa y, en vista de su vinculación con la compra de armas y la financiación del terrorismo, el blanqueo de dinero, las transacciones financieras y el tráfico de drogas; $y$

b) Un Equipo de Apoyo a la Aplicación de las Sanciones, coordinado por el Grupo de Vigilancia, de hasta 15 miembros con experiencia en esferas como aduanas, seguridad fronteriza y medidas contra el terrorismo, desplegados en los Estados a los que se hace referencia en el párrafo 2 supra, en plena consulta y estrecha cooperación con esos Estados, 
5. Pide al Grupo de Vigilancia que presente informes al comité establecido en virtud de la resolución 1267 (1999), incluso mediante reuniones de información para los expertos del mecanismo de vigilancia acerca de la labor de ese mecanismo en virtud del mandato establecido en el párrafo 3 supra, y pide también al Equipo de Apoyo a la Aplicación de las Sanciones que presente informes al Grupo de Vigilancia por lo menos una vez al mes;

6. Pide también al comité establecido en virtud de la resolución 1267 (1999) que presente al Consejo de Seguridad informes periódicos sobre la aplicación de la presente resolución;

7. Pide a todos los Estados, a las Naciones Unidas y a las partes interesadas que cooperen sin reservas ni demoras con el mecanismo de vigilancia,

8. Insta a todos los Estados a que adopten medidas inmediatas para hacer cumplir y reforzar, promulgando leyes o adoptando medidas administrativas, según proceda, las medidas dispuestas en sus leyes o reglamentos nacionales contra sus ciudadanos u otros individuos o entidades que operen en su territorio, para prevenir y castigar violaciones de las medidas impuestas por las resoluciones 1267 (1999) y 1333 (2000) y a que informen al comité establecido en virtud de la resolución 1267 (1999) de la adopción de esas medidas, e invita a los Estados a que comuniquen al comité los resultados de todas las medidas de observancia de la ley y todas las investigaciones conexas, a menos que esto perjudique a las investigaciones o a las medidas de represión;

9. Pide al secretario general que disponga lo necesario para apoyar la labor del mecanismo de vigilancia, como gasto de la organización y con cargo a un Fondo Fiduciario de las Naciones Unidas establecido para este fin, afirma que este Fondo Fiduciario será establecido por el secretario general, alienta a los Estados a que aporten contribuciones al Fondo y a que proporcionen, por conducto del secretario general, personal, equipo y servicios al mecanismo de vigilancia, y pide también al secretario general que mantenga al comité establecido en virtud de la resolución 1267 (1999) informado periódicamente acerca de los arreglos financieros de apoyo al mecanismo;

10. Expresa su intención de examinar la aplicación de las medidas impuestas por las resoluciones 1267 (1999) y 1333 (2000) sobre la base de la información que le proporcione el mecanismo de vigilancia por conducto del comité establecido en virtud de la resolución 1267 (1999);

11. Decide seguir ocupándose de la cuestión. 


\section{RESOLUCIÓN 1368 (2001)}

S/RES/1368 (2001)

Distr. general

12 de septiembre de 2001

Aprobada por el Consejo de Seguridad en su 4370 sesión, celebrada el 12 de septiembre de 2001

El Consejo de Seguridad,

Reafirmando los propósitos y principios de la Carta de las Naciones Unidas. Decidido a combatir por todos los medios las amenazas a la paz y la seguridad internacionales creadas por actos de terrorismo,

Reconociendo el derecho inmanente a la legítima defensa individual o colectiva va de conformidad con la Carta de las Naciones Unidas,

1. Condena inequívocamente en los términos más enérgicos los horrendos ataques terroristas que tuvieron lugar el 11 de septiembre de 2001 en Nueva York, Washington, D. C. y Pennsylvania y considera que esos actos, al igual que cualquier acto de terrorismo internacional, constituyen una amenaza para la paz y la seguridad internacionales;

2. Expresa su más sentido pésame y sus más profundas condolencias a las víctimas y sus familias, así como al pueblo y el gobierno de los Estados Unidos de América,

3. Insta a todos los Estados a que colaboren con urgencia para someter a la acción de la justicia a los autores, organizadores y patrocinadores de estos ataques terroristas y subraya que los cómplices de los autores, organizadores y patrocinadores de estos actos y los responsables de darles apoyo o asilo tendrán que rendir cuenta de sus hechos;

4. Exhorta a la comunidad internacional a que redoble sus esfuerzos por prevenir y reprimir los actos de terrorismo, entre otras cosas cooperando más y cumpliendo plenamente los convenios internacionales contra el terrorismo que sean pertinentes y las resoluciones del Consejo de Seguridad, en particular la resolución 1269 (1999), de 19 de octubre de 1999;

5. Expresa que está dispuesto a tomar todas las medidas que sean necesarias para responder a los ataques terroristas perpetrados el 11 de septiembre de 2001 y para combatir el terrorismo en todas sus formas, con arreglo a las funciones que le incumben en virtud de la Carta de las Naciones Unidas;

6. Decide seguir ocupándose de la cuestión. 
RESOLUCIÓN 1373 (2001)

Distr. general

28 de septiembre de 2001

Aprobada por el Consejo de Seguridad en su 4385 sesión, celebrada el 28 de septiembre de 2001

El Consejo de Seguridad,

Reafirmando sus resoluciones 1269 (1999) de 19 de octubre de 1999 y 1368 (2001) de 12 de septiembre de 2001,

Reafirmando también su condena inequívoca de los ataques terroristas ocurridos en Nueva York, Washington, D. C., y Pennsylvania el 11 de septiembre de 2001, y expresando su determinación de prevenir todos los actos de esa índole,

Reafirmando asimismo que esos actos, al igual que todo acto de terrorismo internacional, constituyen una amenaza a la paz y la seguridad internacionales,

Reafirmando el derecho inmanente de legítima defensa individual o colectiva reconocido en la Carta de las Naciones Unidas y confirmado en la resolución 1368 (2001),

Reafirmando la necesidad de luchar con todos los medios, de conformidad con la Carta de las Naciones Unidas, contra las amenazas a la paz y la seguridad internacionales representadas por los actos de terrorismo,

Profundamente preocupado por el aumento, en varias regiones del mundo, de actos de terrorismo motivados por la intolerancia o el extremismo,

Insta a los Estados a trabajar de consuno urgentemente para prevenir y reprimir los actos de terrorismo, en particular acrecentando su cooperación y cumpliendo plenamente los convenios internacionales contra el terrorismo que sean pertinentes,

Reconociendo la necesidad de que los Estados complementen la cooperación internacional adoptando otras medidas para prevenir y reprimir en sus territorios, por todos los medios legales, la financiación y preparación de esos actos de terrorismo,

Reafirmando el principio establecido por la Asamblea General en su declaración de octubre de 1970 (2625) (XXV) y confirmado por el Consejo de Seguridad en su resolución 1189 (1998), de 13 de agosto de 1998, a saber, que todos los Estados tienen el deber de abstenerse de organizar, instigar y apoyar actos terroristas perpetrados en otro Estado o de participar en ellos, y de permitir actividades organizadas en su territorio encaminadas a la comisión de dichos actos, 
Actuando en virtud del capítulo VII de la Carta de las Naciones Unidas,

1. Decide que todos los Estados:

a) Prevengan y repriman la financiación de los actos de terrorismo;

b) Tipifiquen como delito la provisión o recaudación intencionales, por cualesquiera medios, directa o indirectamente, de fondos por sus nacionales o en sus territorios con intención de que dichos fondos se utilicen, o con conocimiento de que dichos fondos se utilizarán, para perpetrar actos de terrorismo;

c) Congelen sin dilación los fondos y demás activos financieros o recursos económicos de las personas que cometan, o intenten cometer, actos de terrorismo o participen en ellos o faciliten su comisión; de las entidades de propiedad o bajo el control, directos o indirectos, de esas personas, y de las personas y entidades que actúen en nombre de esas personas y entidades o bajo sus órdenes, inclusive los fondos obtenidos o derivados de los bienes de propiedad o bajo el control, directos o indirectos, de esas personas y de las personas y entidades asociadas con ellos;

d) Prohíban a sus nacionales o a todas las personas y entidades en sus territorios que pongan cualesquiera fondos, recursos financieros o económicos o servicios financieros o servicios conexos de otra índole, directa o indirectamente, a disposición de las personas que cometan o intenten cometer actos de terrorismo o faciliten su comisión o participen en ella, de las entidades de propiedad o bajo el control, directos o indirectos, de esas personas y de las personas y entidades que actúen en nombre de esas personas o bajo sus órdenes;

2. Decide también que todos los Estados:

a) Se abstengan de proporcionar todo tipo de apoyo, activo o pasivo, a las entidades o personas que participen en la comisión de actos de terrorismo, inclusive reprimiendo el reclutamiento de miembros de grupos terroristas y eliminando el abastecimiento de armas a los terroristas;

b) Adopten las medidas necesarias para prevenir la comisión de actos de terrorismo, inclusive mediante la provisión de alerta temprana a otros Estados mediante el intercambio de información;

c) Denieguen refugio a quienes financian, planifican o cometen actos de terrorismo, o prestan apoyo a esos actos, o proporcionan refugios;

d) Impidan que quienes financian, planifican, facilitan o cometen actos de terrorismo utilicen sus territorios respectivos para esos fines, en contra de otros Estados o de sus ciudadanos;

e) Aseguren el enjuiciamiento de toda persona que participe en la financiación, planificación, preparación o comisión de actos de terrorismo o preste apoyo a esos actos, y aseguren que, además de cualesquiera otras medidas de represión de esos actos que se adopten, dichos actos de terrorismo queden tipificados como delitos graves en las leyes y otros instrumen- 
tos legislativos internos y que el castigo que se imponga corresponda a la gravedad de esos actos de terrorismo;

f) Se proporcionen recíprocamente el máximo nivel de asistencia en lo que se refiere a las investigaciones o los procedimientos penales relacionados con la financiación de los actos de terrorismo o el apoyo prestado a éstos, inclusive por lo que respecta a la asistencia para la obtención de las pruebas que posean y que sean necesarias en esos procedimientos;

g) Impidan la circulación de terroristas o de grupos terroristas mediante controles eficaces en frontera y controles de la emisión de documentos de identidad y de viaje, y mediante la adopción de medidas para evitar la falsificación, la alteración ilegal y la utilización fraudulenta de documentos de identidad y de viaje;

3. Exhorta a todos los Estados a:

a) Encontrar medios para intensificar y agilizar el intercambio de información operacional, especialmente en relación con las actividades o movimientos de terroristas individuales o de redes de terroristas; los documentos de viaje alterados ilegalmente o falsificados; el tráfico de armas, explosivos o materiales peligrosos; la utilización de tecnologías de las comunicaciones por grupos terroristas y la amenaza representada por la posesión de armas de destrucción en masa por parte de grupos terroristas;

b) Intercambiar información de conformidad con el derecho internacional y la legislación interna y cooperar en las esferas administrativas y judiciales para impedir la comisión de actos de terrorismo;

e) Cooperar, en particular mediante acuerdos y convenios bilaterales y multilaterales, para impedir y reprimir los ataques terroristas, y adoptar medidas contra quienes cometan esos actos;

d) Adherirse tan pronto como sea posible a los convenios y protocolos internacionales pertinentes relativos al terrorismo, inclusive el Convenio Internacional para la represión de la financiación del terrorismo, de 9 de diciembre de 1999;

e) Fomentar la cooperación y aplicar plenamente los convenios y protocolos internacionales pertinentes relativos al terrorismo, así como las resoluciones del Consejo de Seguridad 1269 (1999) y 1368 (2001);

f) Adoptar las medidas apropiadas de conformidad con las disposiciones pertinentes de la legislación nacional y el derecho internacional, inclusive las normas internacionales en materia de derechos humanos, antes de conceder el estatuto de refugiado, con el propósito de asegurarse de que el solicitante de asilo no haya planificado o facilitado actos de terrorismo ni participado en su comisión;

g) Asegurar, de conformidad con el derecho internacional, que el estatuto de refugiado no sea utilizado de modo ilegítimo por los autores, organizadores o patrocinadores de los actos de terrorismo, y que no se reconozca 
la reivindicación de motivaciones políticas como causa de denegación de las solicitudes de extradición de presuntos terroristas;

4. Observa con preocupación la conexión estrecha que existe entre el terrorismo internacional y la delincuencia transnacional organizada, las drogas ilícitas, el blanqueo de dinero, el tráfico ilícito de armas y la circulación ilícita de materiales nucleares, químicos, biológicos y otros materiales potencialmente letales, y a ese respecto pone de relieve la necesidad de promover la coordinación de las iniciativas en los planos nacional, subregional, regional e internacional, para reforzar la respuesta internacional a este reto y amenaza graves a la seguridad internacional;

5. Declara que los actos, métodos y prácticas terroristas son contrarios a los propósitos y principios de las Naciones Unidas y que financiar intencionalmente actos de terrorismo, planificarlos e incitar a su comisión también es contrario a dichos propósitos y principios de las Naciones Unidas;

6. Decide establecer, de conformidad con el artículo 28 de su reglamento provisional, un Comité del Consejo de Seguridad integrado por todos los miembros del consejo, para verificar la aplicación de la presente resolución, con la asistencia de los expertos que se consideren apropiados, y exhorta a todos los Estados a que informen al comité, a más tardar 90 días después de la fecha de aprobación de la resolución y con posterioridad conforme a un calendario que será propuesto por el comité, de las medidas que hayan adoptado para aplicar la presente resolución;

7. Pide al comité que establezca sus tareas, presente un programa de trabajo en el plazo de 30 días después de la aprobación de la presente resolución y determine el apoyo que necesita, en consulta con el secretario general;

8. Expresa su determinación de adoptar todas las medidas necesarias para asegurar la aplicación plena de la presente resolución de conformidad con las funciones que se le asignan en la carta;

9. Decide seguir ocupándose de la cuestión.

\section{ORGANIZACIÓN DE ESTADOS AMERICANOS. VIGÉSIMO CUARTA REUNIÓN DE Consulta DE Ministros de Relaciones EXTERIORES}

OEA/Ser.F/II.24RC.24/RES.1/01

21 de septiembre 2001

Washington, D. C. Original: portugués

AMENAZA TERRORISTA EN LAS AMÉRICAS

Resolución aprobada en la primera sesión plenaria, celebrada el 21 de septiembre de 2001 sujeta a revisión por la Comisión de Estilo 
La Vigésimo Cuarta Reunión de Consulta de Ministros de Relaciones Exteriores, actuando como órgano de consulta en aplicación del Tratado Interamericano de Asistencia Recíproca (TIAR),

CONSIDERANDO los ataques terroristas perpetrados contra personas inocentes de distintos países que tuvieron lugar el 11 de septiembre de 2001 en el territorio de los Estados Unidos de América;

RECORDANDO el derecho inmanente de los Estados de actuar en ejercicio del derecho de legítima defensa, individual y colectiva, de conformidad con la Carta de las Naciones Unidas y el Tratado Interamericano de Asistencia Recíproca (TIAR);

DESTACANDO que el artículo 2 de la Carta de la Organización de los Estados Americanos proclama que uno de los propósitos esenciales de la organización es fortalecer la paz y la seguridad del Continente y organizar la acción solidaria en caso de agresión;

CONSIDERANDO que la obligación de asistencia mutua y defensa colectiva de las repúblicas americanas se vincula esencialmente con sus ideales democráticos y su voluntad de cooperar permanentemente en el cumplimiento de los principios y propósitos de una política de paz; y

TOMANDO NOTA de la resolución CP/RES. 797 (1293/01), del 19 de septiembre de 2001, aprobada por el Consejo Permanente de la Organización de los Estados Americanos actuando provisionalmente como rgano de Consulta del Tratado Interamericano de Asistencia Recíproca (TIAR), en la cual se convocó una Reunión de Ministros de Relaciones Exteriores para actuar como rgano de Consulta en aplicación del TIAR, con relación a los ataques terroristas perpetrados en los Estados Unidos de América, el 11 de septiembre de 2001,

\section{RESUELVE:}

Estos ataques terroristas contra los Estados Unidos de América son ataques contra todos los Estados americanos y, de conformidad con todas las disposiciones pertinentes del Tratado Interamericano de Asistencia Recíproca (TIAR) y el principio de solidaridad continental, todos los Estados partes del Tratado de Río deberán brindar asistencia recíproca efectiva para enfrentar tales ataques y la amenaza de ataques similares contra cualquier Estado americano, y para mantener la paz y la seguridad del Continente.

Si un Estado parte tiene elementos fundados para presumir que alguna persona en su territorio pueda haber participado o prestado cualquier asistencia en los ataques perpetrados el 11 de septiembre de 2001, que esté protegiendo a los perpetradores o que pueda estar involucrada en actividades terroristas, ese Estado parte deberá utilizar todas las medidas disponibles conforme a la ley para perseguir, capturar, extraditar y castigar a estos individuos. 
Los Estados partes prestarán asistencia y apoyo adicional a Estados Unidos de América y entre sí, según corresponda, en relación con los ataques del 11 de septiembre y con el fin de prevenir futuros actos terroristas.

Los Estados partes mantendrán al Órgano de Consulta debidamente informado sobre todas las medidas adoptadas de conformidad con esta resolución.

Mantener abierta la Vigésimo Cuarta Reunión de Consulta de Ministros de Relaciones Exteriores, actuando como Órgano de Consulta, con el fin de asegurar la pronta y efectiva aplicación de esta resolución y, si fuere necesario, adoptar las medidas adicionales adecuadas para abordar este asunto.

Designar una comisión integrada por los representantes de cada Estado parte del Tratado de Río en el Consejo Permanente de la OEA, con el fin de realizar consultas adicionales y adoptar medidas para darle seguimiento a los criterios aquí adoptados.

Solicitar a todos los gobiernos americanos y a la Organización de los Estados Americanos que presten su total cooperación en la aplicación de esta resolución.

Encomendar al Consejo Permanente que tome las medidas pertinentes en cumplimiento de la Resolución RC.23/doc.7/01 adoptadas en la Vigésimo Tercera Reunión de Consulta de Ministros de Relaciones Exteriores.

Informar de inmediato al Consejo de Seguridad de las Naciones Unidas sobre el texto de la presente resolución y sobre cualquier decisión que pueda adoptarse con relación a este asunto.

\section{VigÉSIMO TERCERA REUNIÓN DE CONSULTA DE MINISTROS DE RELACIONES EXTERIORES}

OEA/Ser.F/II.23

RC.23/RES.1/01

21 septiembre 2001

Washington, D.C. Original: español

\section{FORTALECIMIENTO DE LA COOPERACIÓN HEMISFÉRICA PARA PREVENIR, COMBATIR Y ELIMINAR EL TERRORISMO}

Resolución aprobada en la primera sesión plenaria, celebrada el 21 de septiembre de 2001; sujeta a revisión por la Comisión de Estilo

La Reunión de Consulta de Ministros de Relaciones Exteriores de los Estados Miembros de la Organización de los Estados Americanos,

LAMENTANDO PROFUNDAMENTE los ataques terroristas perpetrados contra personas inocentes de muchas naciones que tuvieron lugar en el territorio de los Estados Unidos de América el 11 de septiembre de 2001; 
REAFIRMANDO los principios y disposiciones contenidos en la Carta de la Organización de los Estados Americanos y la Carta de las Naciones Unidas;

RECORDANDO las resoluciones adoptadas en el marco del sistema interamericano de seguridad hemisférica y lucha contra el terrorismo;

TENIENDO EN CUENTA las resoluciones de la Asamblea General de las Naciones Unidas y del Consejo de Seguridad A/RES/56/1 y S/RES/1368 (2201) del 12 de septiembre de 2001, mediante las cuales las Naciones Unidas condenaron enérgicamente los ataques terroristas perpetrados en los Estados Unidos de América y exhortaron a todos los Estados a colaborar con urgencia para someter a la justicia a los autores, organizadores y patrocinadores de estos actos y a redoblar sus esfuerzos para prevenir y reprimir los actos de terrorismo, así como todas las resoluciones de la Asamblea General y el Consejo de Seguridad sobre los medios para prevenir, combatir y eliminar el terrorismo internacional;

CONSIDERANDO la declaración aprobada el 11 de septiembre de 2001 por la Asamblea General de la Organización de los Estados Americanos en su vigésimo octavo periodo extraordinario de sesiones, la cual condenó en los más enérgicos términos los actos terroristas perpetrados en Estados Unidos de América, que demostraron la necesidad de fortalecer la cooperación hemisférica para combatir este flagelo y su plena solidaridad con el pueblo y el gobierno de los Estados Unidos de América;

TENIENDO EN CUENTA ADEMÁs que el secretario general de las Naciones Unidas declaró el 12 de septiembre de 2001 que todas las naciones del mundo deben unirse solidariamente con las víctimas del terrorismo y en su determinación de tomar acción, tanto contra los propios terroristas como contra todos quienes les ofrecen cualquier tipo de refugio, asistencia o apoyo;

TENIENDO PRESENTE la Convención para Prevenir y Sancionar los Actos de Terrorismo Configurados como Delitos contra las Personas y la Extorsión Conexa cuando éstos tengan trascendencia internacional;

RECORDANDo la Declaración de Principios de las Cumbres de las Américas de Miami, Santiago y la ciudad de Quebec;

Teniendo en Cuenta la Declaración de Lima para Prevenir, Combatir y Eliminar el Terrorismo y el Plan de Acción de Cooperación Hemisférica para Prevenir, Combatir y Eliminar el Terrorismo, adoptado en el marco de la Primera Conferencia Especializada Interamericana sobre Terrorismo en Lima, Perú, en abril de 1996, así como el Compromiso de Mar del Plata, adoptado en la Segunda Conferencia Especializada Interamericana sobre Terrorismo que propuso el establecimiento del Comité Interamericano Contra el Terrorismo (Cicte) y proporcionó los Lineamientos para la Cooperación Interamericana frente a Actos y Actividades Terroristas y las Medidas para Eliminar la Captación de Fondos para el Terrorismo, 
TENIENDO PRESENTE que mediante la resolución AG/RES. 1650 (XXXIX-O/99) la Asamblea General estableció el Cicte;

REAFIRMANDO el rechazo absoluto de los pueblos y los gobiernos de las Américas a los actos y actividades terroristas que atentan contra la democracia y la seguridad de los Estados del Hemisferio;

RECONOCIENDO el derecho inmanente a la legítima defensa individual y colectiva de conformidad con las Cartas de la Organización de los Estados Americanos y de las Naciones Unidas;

CONVENCIDOS de que la respuesta de los Estados miembros ante la situación actual exige que se apliquen o adopten, de acuerdo con su legislación nacional, medidas urgentes, a nivel nacional e internacional, para combatir las amenazas a la paz, la democracia y la seguridad del Hemisferio a raíz de los actos terroristas y que se someta a la justicia a los autores, organizadores y patrocinadores de estos actos, así como a quienes les brinden asistencia, apoyo o protección;

CONSIDERANDO también que la Carta Democrática Interamericana, adoptada por la Asamblea General en su vigésimo octavo periodo extraordinario de sesiones, el 11 de septiembre de 2001 reconoce el compromiso de los Estados miembros de promover y defender la democracia, y que ningún Estado democrático puede permanecer indiferente a la clara amenaza que el terrorismo representa para las instituciones y libertades democráticas;

RECONOCIENDO ADEMÁs que los actos terroristas, y el clima de inseguridad que generan, tienen efectos altamente perjudiciales para el comercio internacional, la industria del turismo y el mantenimiento de flujos de capital para la inversión, y que también se constituyen en una amenaza a la estabilidad económica y financiera, el progreso y la paz social en los países de nuestro Hemisferio;

CONSCIENTES de que las nuevas amenazas a la seguridad hemisférica deben ser consideradas por la OEA de forma congruente con las decisiones adoptadas por las Naciones Unidas, en particular la resolución 1368 del Consejo de Seguridad y la resolución A/RES/56/1 de la Asamblea General de las Naciones Unidas.

TOMANDO EN CUENTA la responsabilidad de todos los Estados de cooperar en la identificación, juicio y sanción de todos los responsables de los actos terroristas, que constituyen crímenes de la mayor gravedad y la necesidad imperativa de acelerar los procesos de extradición, en los casos que ésta procede;

RESUELVE:

Condenar enérgicamente los ataques terroristas perpetrados en el territorio de los Estados Unidos de América el 11 de septiembre de 2001. 
Expresar sus más sentidas condolencias y solidaridad con el pueblo y el gobierno de los Estados Unidos de América y, en particular, con las familias de las víctimas de este abominable crimen.

Exhortar a todos los Estados miembros y a toda la comunidad internacional a adoptar medidas eficaces para impedir que los grupos terroristas tengan capacidad de operar en sus territorios, haciendo notar que los responsables de dar ayuda, apoyo o protección a los autores, organizadores y patrocinadores de estos actos son igualmente cómplices de éstos.

Exhortar a todos los Estados a reforzar la cooperación, en los planos regional e internacional, para perseguir, capturar, enjuiciar, sancionar y, cuando corresponda, acelerar la extradición de los perpetradores, organizadores y patrocinadores de actos terroristas, así como fortalecer la cooperación judicial recíproca y el intercambio oportuno de información.

Reafirmar que la lucha contra el terrorismo debe realizarse con pleno respeto a la ley, a los derechos humanos y a las instituciones democráticas, para preservar el estado de derecho, las libertades y los valores democráticos en el Hemisferio.

Exhortar a todos los Estados miembros a promover la más amplia tolerancia y la convivencia armónica en sus sociedades, en reconocimiento de la diversidad racial, cultural, étnica y religiosa de las comunidades que componen nuestro Hemisferio y cuyos derechos y libertades fundamentales fueron recientemente reafirmados en la Carta Democrática Interamericana.

Instar a los Estados que no lo hayan hecho, a suscribir o ratificar, según sea el caso, la Convención Internacional para la Supresión del Financiamiento del Terrorismo, adoptada en Nueva York el 9 de diciembre de 1999.

Instruir al Consejo Permanente para que convoque lo antes posible una reunión del Comité Interamericano contra el Terrorismo, con el fin de que identifique acciones urgentes dirigidas a fortalecer la cooperación interamericana para prevenir, combatir y eliminar el terrorismo en el Hemisferio.

Encomendar al Consejo Permanente la elaboración de un proyecto de Convención Interamericana contra el Terrorismo, con miras a presentarlo a la próxima Asamblea General de la organización. Asimismo, instar a los Estados a estudiar la repercusión jurídica internacional de la conducta de las autoridades gubernamentales que apoyan con financiamiento, protección o amparo a personas y grupos terroristas.

Encomendar a la Comisión de Seguridad Hemisférica que acelere sus labores con miras a la Conferencia Especial sobre Seguridad, tomando en cuenta los aportes del CICTE, y formule recomendaciones específicas al Consejo Permanente. 
Encomendar al secretario general que preste la asistencia necesaria a la actividad del CICTE de conformidad con la resolución AG/RES. 1650 (XXIX-O/99).

Invitar a la Junta Interamericana de Defensa a que preste la asesoría necesaria a la Comisión de Seguridad Hemisférica, de conformidad con la resolución AG/RES. 1240 (XXIII-O/93) y cuando la Comisión así lo solicite.

\section{NATO. STATEMENT By THE NORTH ATLANTIC COUNCIL}

On September 12th, the North Atlantic Council met again in response to the appalling attacks perpetrated yesterday against the United States.

The Council agreed that if it is determined that this attack was directed from abroad against the United States, it shall be regarded as an action covered by Hyperlink http://www.nato.int/docu/basictxt/treaty.htm V Art05 Article 5 of the Washington Treaty, which states that an armed attack against one or more of the Allies in Europe or North America shall be considered an attack against them all.

The commitment to collective self-defence embodied in the Washington Treaty was first entered into in circumstances very different from those that exist now, but it remains no less valid and no less essential today, in a world subject to the scourge of international terrorism. When the Heads of State and Government of NATO met in Washington in 1999, they paid tribute to the success of the Alliance in ensuring the freedom of its members during the Cold War and in making possible a Europe that was whole and free. But they also recognised the existence of a wide variety of risks to security, some of them quite unlike those that had called NATO into existence. More specifically, they condemned terrorism as a serious threat to peace and stability and reaffirmed their determination to combat it in accordance with their commitments to one another, their international commitments and national legislation.

Article 5 of the Washington Treaty stipulates that in the event of attacks falling within its purview, each Ally will assist the Party that has been attacked by taking such action as it deems necessary. Accordingly, the United States' NATO Allies stand ready to provide the assistance that may be required as a consequence of these acts of barbarism.

Article 5: The Parties agree that an armed attack against one or more of them in Europe or North America shall be considered an attack against them all and consequently they agree that, if such an armed attack occurs, each of them, in exercise of the right of individual or collective selfdefence recognised by Article 51 of the Charter of the United Nations, will 
assist the Party or Parties so attacked by taking forthwith, individually and in concert with the other Parties, such action as it deems necessary, including the use of armed force, to restore and maintain the security of the North Atlantic area.

Any such armed attack and all measures taken as a result thereof shall immediately be reported to the Security Council. Such measures shall be terminated when the Security Council has taken the measures necessary to restore and maintain international peace and security.

Article 51 of the Charter of the United Nations: Nothing in the present Charter shall impair the inherent right of individual or collective self-defence if an armed attack occurs against a Member of the United Nations, until the Security Council has taken measures necessary to maintain international peace and security. Measures taken by Members in the exercise of this right of self-defence shall be immediately reported to the Security Council and shall not in any way affect the authority and responsibility of the Security Council under the present Charter to take at any time such action as it deems necessary in order to maintain or restore international peace and security.

\section{Statement By The North AtLANTiC CounciL}

The North Atlantic Council met tonight to express its solidarity with the United States of America at this moment of great tragedy and mourning. Our deepest sympathy lies with the victims, their families and all Americans. The NATO nations unanimously condemn these barbaric acts committed against a NATO member state. The mindless slaughter of so many innocent civilians is an unacceptable act of violence without precedent in the modern era. It underscores the urgency of intensifying the battle against terrorism, a battle that the NATO countries - indeed all civilised nationsmust win. All Allies stand united in their determination to combat this scourge.

At this critical moment, the United States can rely on its 18 Allies in North America and Europe for assistance and support. NATO solidarity remains the essence of our Alliance. Our message to the people of the United States is that we are with you. Our message to those who perpetrated these unspeakable crimes is equally clear: you will not get away with it. 


\section{STATEMENT BY THE SECRETARY GENERAL OF NATO LORD ROBERTSON}

I condemn in the strongest possible terms the senseless attacks which have just been perpetrated against the United States of America. My sympathies go to the American people, the victims and their families. These barbaric acts constitute intolerable aggression against democracy and underline the need for the international community and the members of the Alliance to unite their forces in fighting the scourge of terrorism.

\section{STATEMENT By The EURO-ATLANTiC PARTNERSHIP COUNCIL}

We, the member nations of the Euro-Atlantic Partnership Council, gathered today to express our solidarity with the people of the United States of America following yesterday's tragic events. These brutal and senseless atrocities have caused suffering on a massive scale. Our deepest sympathies go to the victims and their families.

We are appalled by these barbaric acts and condemn them unconditionally. These acts were an attack on our common values. We will not allow these values to be compromised by those who follow the path of violence. We pledge to undertake all efforts to combat the scourge of terrorism. We stand united in our belief that the ideals of partnership and cooperation will prevail.

EAPC Member Countries are: Albania, Armenia, Austria, Azerbaijan, Belarus, Belgium, Bulgaria, Canada, Croatia, Czech Republic, Denmark, Estonia, Finland, France, Georgia, Germany, Greece, Hungary, Iceland, Ireland, Italy, Kazakhstan, Kyrghyz Republic, Latvia, Lithuania, Luxembourg, Moldova, the Netherlands, Norway, Poland, Portugal, Romania, Russia, Slovakia, Slovenia, Spain, Sweden, Switzerland, the former Yugoslav Republic of Macedonia ( Hyperlink “http://www.nato.int/docu/pr/2001/' 1 "FN2" 2), Tadjikistan, Turkey, Turkmenistan, Ukraine, United Kingdom, United States, Uzbekistan.

Turkey recognises the Republic of Macedonia with its constitutional name. 


\section{PRESS STATEMENT. MEETING IN EXTRAORDINARY SESSION OF THE NATO-RUSSIA PERMANENT JOINT COUNCIL AT AMBASSADORIAL LEVEL}

The NATO-Russia Permanent Joint Council, meeting in extraordinary session at Ambassadorial level on 13 September 2001, expressed its anger and indignation at the barbaric acts committed against the people of the United States of America. The deepest sympathies of Allies and Russia lie with the victims and their families. While Allies and Russia have suffered from terrorist attacks against civilians, the horrific scale of the attacks of 11 September is without precedent in modern history. NATO and Russia are united in their resolve not to let those responsible for such an inhuman act to go unpunished. NATO and Russia call on the entire international community to unite in the struggle against terrorism.

NATO and Russia will intensify their cooperation under the Founding Act to defeat this scourge.

\section{PRESIDENCIA DE LA REPÚBLICA}

Palacio Nacional, 7 de octubre de 2001.

Versión estenográfica del mensaje que el presidente Vicente Fox Quesada dirigió a la nación esta noche, desde el despacho presidencial de Palacio Nacional

Hola, muy buenas noches, mexicanos y mexicanas.

El día de hoy ha dado inicio una operación internacional conjunta, encabezada por Estados Unidos y el Reino Unido en el territorio de Afganistán, cuyo objetivo manifestado consiste en ubicar, detener y llevar ante la acción de la justicia a los miembros de las organizaciones terroristas que el pasado 11 de septiembre perpetraron ataques sobre la población civil con aviones comerciales, en las ciudades de Nueva York y Washington, dando como resultado una muy lamentable pérdida de vidas humanas, incluyendo las de un número incierto de mexicanos y daños materiales incalculables.

El gobierno de México ha recibido seguridades — de quienes encabezan la operación- de que ésta no se dirige en contra de la población civil o contra credo, ideología o religión alguna. Se concentra en combatir el flagelo del terrorismo en todas sus manifestaciones. 
Es por ello que el gobierno de México apoya con firmeza estas acciones.

La comunidad internacional, a través de organismos de alcance universal como el Consejo de Seguridad de la Organización de las Naciones Unidas, y de carácter regional como la Organización de Estados Americanos, han expresado su más profundo y enérgico rechazo al terrorismo.

México, como miembro de estos foros y preocupado por el mantenimiento de la paz y la seguridad internacionales, se ha sumado a esta condena universal en contra de este grave crimen.

México reitera su más firme respaldo a las resoluciones 1368 y 1373 del Consejo de Seguridad de las Naciones Unidas, con el fin de combatir el terrorismo como una amenaza a la paz y la seguridad internacionales; de someter a los terroristas a la acción de la justicia y de procurar que nunca más se recurra a estas prácticas de lesa humanidad.

Las resoluciones emitidas por el Consejo de Seguridad de las Naciones Unidas obligan a todos los estados miembros a cooperar para identificar y someter a la justicia a los responsables directos o indirectos de estos actos; a desmantelar sus bases de operación, sus mecanismos de financiamiento, sus redes clandestinas y a prevenir nuevos atentados.

El terrorismo no reconoce fronteras, no selecciona víctimas y a nadie respeta. Por ello México asume que los ataques terroristas del 11 de septiembre fueron un ataque contra la humanidad y, por tanto, atentan también contra el interés nacional, la tranquilidad y las aspiraciones de desarrollo de los y las mexicanas.

Es por ello que nuestras acciones están enfocadas al intercambio de inteligencia, procuración de justicia, prevención, protección a instalaciones, resguardo de las fronteras y vigilancia migratoria, entre otras.

La comunidad internacional no desea una nueva guerra, tampoco México.

El atentado brutal del 11 de septiembre ha desatado este conflicto y hace de la lucha contra el terrorismo un imperativo para la seguridad nacional.

El gobierno de México reitera su firme voluntad de combatir el terrorismo en todas sus formas y manifestaciones, dentro del marco constitucional, de las convenciones relevantes en la materia y del derecho internacional.

Desearía — por mi parte - dirigir un mensaje muy particular a los mexicanos y mexicanas.

Como presidente de la república estaré en contacto permanente con el Congreso de la Unión, con los partidos políticos y la sociedad civil, para construir y consolidar el más amplio consenso entre los mexicanos.

El gobierno de la república viene realizando todas las acciones a su alcance, para garantizar la seguridad y la tranquilidad de todas las familias mexicanas. 
México está en paz y unido. Las bases de su economía son sólidas. México — como parte de la comunidad internacional— no puede, ni debe evadir su responsabilidad ante la barbarie del terrorismo.

El gobierno de la república está plenamente consciente de su deber de defender, ante todo, la seguridad, la paz y el progreso del pueblo de México. Muy buenas noches. 